\title{
Fluctuations and criticality (dedicated to Reinhard Folk on his 60th birthday)
}

It is our pleasure and honour to present a collection of papers dedicated to Reinhard Folk on the occasion of his 60th birthday. With this Festschrift, colleagues and friends of the jubilee pay tribute to his long lasting and fruitful work in the field of condensed matter physics. His research includes ferroelectric and ferromagnetic phase transitions in regular and disordered systems, neural networks, superconductivity, critical dynamics - this list can be continued. Those, who work in the above mentioned fields certainly know and often use results obtained by R.F. which are currently turning into scientific Folk-lore. However, those who know him personally, recognizing his important contribution to physics, enjoy marvelous features of his extraordinary personality. Openness, readiness for discussion, erudition, interest in people and concern in their problems, sense of humour - that's what makes communication with him so desirable and pleasant. Moreover, his vivid interest in science on a larger scale and a deep concern in its future lead R.F. to activities in science organizations in Austria and the European Union, to his personal participation in different projects aiming at strengthening collaboration with Central and Eastern European countries. His work in this respect is widely recognized and acknowledged by the scientific community.

Dear Reinhard, may your work in future lead you to new discoveries and solutions of puzzles put to us almost on every step of research, may it give you satisfaction of the achieved results, and may we, your friends, enjoy for many more years the wonderful intellectual atmosphere created by your work and your spirit.

Harald Iro, Yurij Holovatch, Ihor Mryglod and Christian von Ferber Linz-Lviv-Freiburg, 2005 


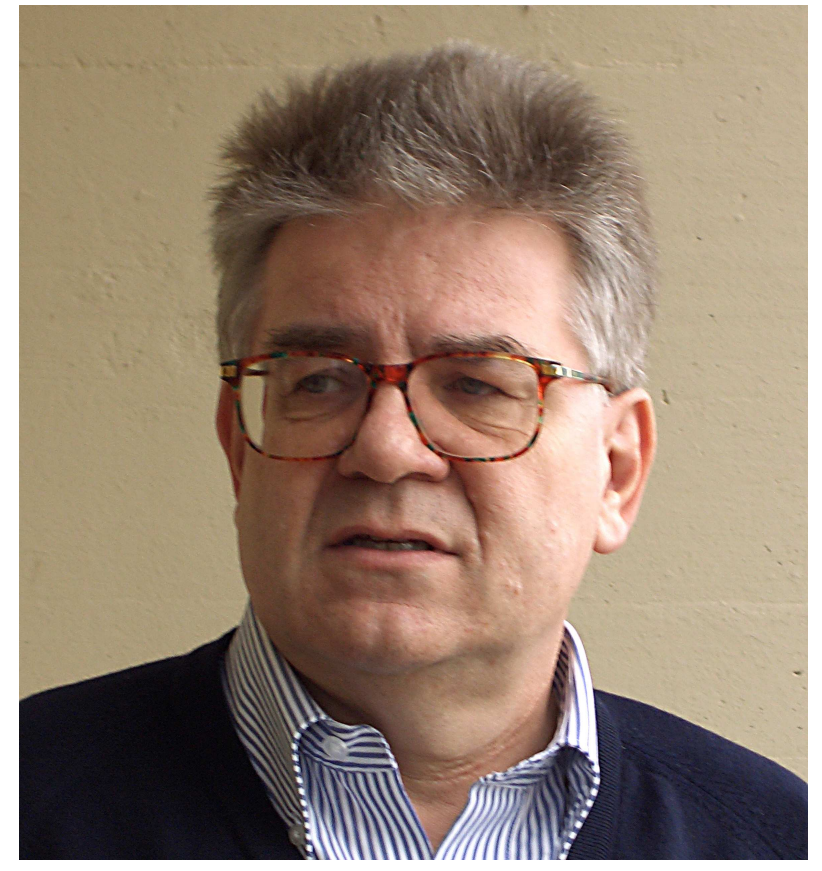

1945, April 29 Born in Neuendettelsau, Germany

1973

1973

1982

1983

1993-

$1995-$

1979-1980

1982

1982-1983

1984

$1986-1987$

1993-2003

1997-1999
Doctoral Thesis at the Institute of Theoretical Physics, University of Vienna

Assistant at the Institute of Theoretical Physics, University of Linz Habilitation in theoretical physics

Permanent position

Senior Associate Professor at the Institute of Theoretical Physics, University of Linz

Member of the Advisor Committee of the Middle European Cooperation on Statistical Physics (MECO)

Scientific visitor at the Institute of Solid State Physics, KFA Jülich, Germany

Walter Schottky Award of the German Physical Society together with Dr. V.Dohm for the work on the critical dynamics in ${ }^{4} \mathrm{He}$

Assistant at the Physics Department of the Technical University München, Germany

Director of the group "Phase Transitions and Critical Phenomena" at the Institute of Theoretical Physics, University of Linz

Visiting Associate Professor (Chair of Prof. Wölfle) at the Physics Department of the Technical University München, Germany Member of the "Bundeskonferenz des wissenschaftlichen und künstlerischen Personals der österreichischen Universitäten und Kunsthochschulen" (BUKO)

Member of the presidency of the BUKO 
1999-2003 Chairman of the presidency of the BUKO

1998- Austrian Delegate in the Technical Committee on Physics of COST

2002- $\quad$ Austrian Delegate in the Steering Committee of the program

"Phase Transitions and Fluctuation Phenomena for Random Dynamics in Spatially Extended Systems" of ESF

\section{References}

1. Folk R., Hydrodynamische Gleichungen dielektrischer Kristalle. Acta Physica Austriaca, 1973, 38, 13.

2. Folk R., Schwabl F., EPR in $\mathrm{SrTiO}_{3}$ dynamical or dirt effect? Solid State Comm., 1974, 15, 37.

3. Petrascheck D., Folk R., Theory of a symmetric LLL interferometer with arbitrary absorption. Phys. Stat. Sol. (a), 1976, 36, 147.

4. Folk R., Iro H., Schwabl F., Elastic phase transitions of second order. Phys. Lett. A, 1976, 57, 112.

5. Folk R., Iro H., Schwabl F., Critical statics of elastic phase transitions. Z. Physik B, 1976, 25, 69 .

6. Folk R., Iro H., Schwabl F., Critical dynamics and statics of uniaxial dipolar magnets. Z. Physik B, 1977, 27, 169.

7. Folk R., Iro H., Schwabl F., Critical dynamics of elastic phase transitions. Phys. Rev. B, 1979, 20, 1229.

8. Folk R., Selke W., Relaxiational models near Lifshitz points. Phys. Lett. A, 1978, 69, 255 .

9. Dohm V., Folk R., Damping of second sound in the critical dynamics of liquid Helium below $T_{\lambda}$. Z. Physik B, 1979, 35, 277; Addendum: Z. Physik B, 1980, 39, 94.

10. Dohm V., Folk R., Nonlinear dynamic renormalization-group analysis above and below the superfluid transition in ${ }^{4}$ He. Phys. Rev. Lett., 1981, 46, 349.

11. Dohm V., Folk R., Nonasymptotic critical dynamics near the superfluid transition in ${ }^{4}$ He. Z. Physik B, 1980, 40, 79.

12. Dohm V., Folk R., Nonlinear renormalization-group analysis applied to the hydrodynamic light scattering spectrum of ${ }^{4} \mathrm{He}$ below $T_{\lambda}$. Z. Physik B, 1981, 41, 251.

13. Dohm V., Folk R. Nonasymptotic critical dynamics of the asymmetric-spin model in two-loop order. Z. Physik B, 1981, 45, 129.

14. Folk R., Kritische Dynamik am suprafluiden Phasenübergang in ${ }^{4}$ He. Acta Physica Austriaca, 1982, 54, 75.

15. Dohm V., Folk R., Critical dynamics near the $\lambda$-transition in ${ }^{4}$ He. Festkörperprobleme (Advances in Solid State Physics), vol. XXII, p. 1, ed. Grosse P. Vieweg, Braunschweig, 1982. 
16. Dohm V., Folk R., Critical dynamics near the $\lambda$-transition of ${ }^{3} \mathrm{He}-{ }^{4} \mathrm{He}$ Mixtures. Phys. Rev. B, 1983, 28, 1332; Erratum: Phys. Rev. B, 1984, 29, 1475.

17. Fishman F., Folk R., Instability of longitudinal driven spin resoncance in superfluid ${ }^{3}$ He-A. Phys. Rev. B, 1984, 29, 6367.

18. Fishman F., Folk R., Instability of nonlinear response of magnetization in superfluid ${ }^{3}$ He-B. Phys. Rev. B, 1984, 30, 1547.

19. Fishman F., Folk R., Stabilizing effect of the static magnetic field and the threshold condition in the logitudinaly driven NMR in superfluid ${ }^{3} \mathrm{He}-\mathrm{B}$. Phys. Rev. B, 1985, 32, 98.

20. Folk R., Iro H., Critical dynamics below the $\lambda$-transition in dilute ${ }^{3} \mathrm{He}-{ }^{4} \mathrm{He}$ mixtures. Phys. Lett. A, 1985, 109, 53.

21. Folk R., Iro H., Paramagnetic neutron scattering and renormalization group theory for isotropic ferromagnets at $T_{\mathrm{c}}$. Phys. Rev. B, 1985, 32, 1880.

22. Moser G., Folk R., Critical behaviour of elastically coupled systems with spatially anisotropic critical fluctuations. SSC, 1986, 57, 707.

23. Folk R., Iro H., Temperature dependence of paramagnetic neutron scattering from Heisenberg ferromagnets above $T_{\mathrm{c}}$. Phys. Rev. B, 1986, 34, 6571.

24. Folk R., Moser G., Nonasymptotic critical behavior of uniaxial systems with strong dipolar interaction. Phys. Lett. A, 1987, 120, 39.

25. Aberger C., Folk R., Shape crossover in the correlation function of isotropic ferromagnets above $T_{\mathrm{c}}$. Phys. Rev. B, 1988, 38, 6693.

26. Aberger C., Folk R., Dynamical crossover to dipolar behaviour in isotropic ferromagnets at and above $T_{\mathrm{c}}$. Phys. Rev. B, 1988, 38, 7207.

27. Kolnberger S., Folk R., Critical fluctuations in superconductors. Phys. Rev. B, 1990, 41, 4083 .

28. Moser G., Folk R., Thermal conductivity near the superfluid transition in ${ }^{3} \mathrm{He}-{ }^{4} \mathrm{He}$ mixtures below $T_{\lambda}$. Phys. Rev. B, 1991, 44, 819.

29. Moser G., Folk R., Critical dynamics in the normal phase near the $\lambda$-transition in ${ }^{3} \mathrm{He}-{ }^{4} \mathrm{He}$ mixtures. I. Theoretical results. J. Low. Temp. Phys., 1992, 86, 57.

30. Moser G., Folk R., Critical dynamics in the normal phase near the $\lambda$-transition in ${ }^{3} \mathrm{He}^{-}{ }^{4} \mathrm{He}$ mixtures. II. Comparison with experiment. J. Low. Temp. Phys., 1992, 86, 99.

31. Folk R., Kartashov A., Lisonek P., Paule P., Symmetries in neural networks: a linear group action approach. J. Phys. A: Math. Gen., 1993, 26, 3195.

32. Folk R., Moser G., Lifshitz points in uniaxial dipolar ferroelectrics. Phys. Rev. B, 1993, 1, No. 47, 13992.

33. Folk R., Moser G., Critical dynamics near the liquid vapor transition in mixtures. Europhys. Lett., 1993, 24, 533.

34. Folk R., Moser G., Critical dynamics in liquids with long-range forces. Phys. Rev. E, 1994, 49, 3128. 
35. Folk R., Kartashov A., A simple elastic model for self-organizing topological mappings. Network: Computation in Neural Systems, 1994, 5, 369.

36. Folk R., Moser G., Universality versus nonuniversality of critical transport properties in liquid mixtures. Int. J. Thermophys., 1995, 16, 1363.

37. Frolov A., Kartashov A., Goltsev A., Folk R., Quality and efficiency of retrieval for Willshaw-like autoassociative networks I: correction. Network: Computation in Neural Systems, 1995, 6, 513.

38. Mryglod I.M., Tokarchuk M.V., Folk R., On the hydrodynamic theory of a magnetic liquid: I. General description. Physica A, 1995, 220, 325.

39. Nasser I., Folk R., Static crossover behavior in the neighborhood of a Lifshitz point. Phys. Rev. B, 1995, 52, 15799.

40. Folk R., Moser G., Nonuniversal dynamical crossover in pure and binary fluids near a critical point. Phys. Rev. Lett., 1995, 75, 2706.

41. Kartashov A., Folk R., Delaunay triangulations in the plane with $O(N \log N)$ storage requirement. Int. J. of Mod. Physics C, 1995, 6, 639.

42. Folk R., Moser G., Transport coefficients in pure fluids and mixtures near a critical point: comparison of the one loop order result with experiment. Condens. Matter Phys., 1996, Iss. 7, 27.

43. Abdel-Hady A., Folk R., Tricritical Lifshitz points in uniaxial ferroelectrics. Phys. Rev. B, 1996, 54, 3851.

44. Folk R., Holovatch Yu., On the critical fluctuations in superconductors. J. Phys. A, 1996, 29, 3409.

45. Folk R., Moser G., Transport properties near a critical point in pure fluids and mixtures. Lectures on Cooperative Phenomena in Condensed Matter Physics, ed. D.I. Uzunov. Heron Press, Sofia, 1996.

46. Kartashov A., Folk R., Topological feature maps by a network of multistate neurons. Neural Network World, 1996, 6, 937.

47. Frolov A., Kartashov A., Goltsev A., Folk R., Quality and efficiency of retrieval for Willshaw-like autoassociative networks III: Potts model. Network: Computation in Neural Systems, 1997, 8, 71.

48. Campbell C.E., R.Folk, Krotscheck E., Critical behavior of ${ }^{4}$ He at negative pressures. J. Low Temp. Phys., 1996, 105, 13.

49. Mryglod I.M., Folk R., On the hydrodynamic theory of a magnetic liquid: II. Hydrodynamic modes in the Heisenberg ferrofluid. Physica A, 1996, 234, 129.

50. Folk R., Moser G., Frequency-dependent shear viscosity, sound velocity and sound attenuation near the critical point in liquids. I. Theoretical results. Phys. Rev. E, 1998, 57, 683 .

51. Nasser I., Abdel-Hady A., Folk R., Specific-heat amplitude ratio near a Lifshitz point. Phys. Rev. B, 1997, 56, 154.

52. Folk R., Holovatch Yu., Is the normal to superconducting phase transition first or 
second order? J. Phys. Stud., 1997, 1, No. 3, 343.

53. Folk R., Moser G., Frequency-dependent shear viscosity, sound velocity and sound attenuation near the critical point in liquids. II. Comparison with experiment. Phys. Rev. E, 1998, 57, 705.

54. Folk R., Moser G., Non-asymptotic transport properties in fluids and mixtures near a critical point. Int. J. Thermophys., 1998, 19, 1003.

55. Folk R., Multicritical behavior in ferroelectrics. Phase Transitions, 1999, 67, 645.

56. Folk R., Moser G., Critical sound in mixtures. Europhys. Lett., 1998, 41, 177.

57. Folk R., Holovatch Yu., Critical fluctuations in normal-to-superconducting transitions. "Correlations, Coherence, and Order", ed by D.V. Shopova, D.I. Uzunov. Kluwer Academic Pub., 1999.

58. Schinagl F., Iro H., Folk R., Magnetic fluids in an external field. European Phys. Journ. B, 1999, 8, 113.

59. Folk R., Holovatch Yu., Yavors'kii T., Five-loop critical exponents of the weakly diluted Ising model: 3d approach versus square root of epsilon expansion. J. Phys. Stud., 1998, 2, No. 2, 213.

60. Folk R., Moser G., Critical dynamics in mixtures. Phys. Rev. E, 1998, 58, 6246.

61. Schinagl F., Iro H., Folk R., Multicritical behaviour in magnetic fluids. Condens. Matter Phys., 1999, 2, 313.

62. Folk R., Moser G., Critical sound in fluids and mixtures. Condens. Matter Phys., 1999, 2, 243.

63. Flossmann G., Folk R., Moser G., Frequency dependent shear viscosity, sound velocity and sound attenuation near the critical point in liquids. III. The shear viscosity. Phys. Rev. E, 1999, 60, 779.

64. Mryglod I., Folk R., Dubyk S., Rudavskii Yu., Dynamic structure factor of a Heisenberg model fluid. Condens. Matter Phys., 1999, 2, 221.

65. Folk R., Holovatch Yu., Yavors'kii T., The correction-to-scaling exponent in dilute systems. JEPT Lett., 1999, 69, 747; Pis'ma ZhETF, 1999, 69, 698.

66. Mryglod I., Folk R., Dubyk S., Rudavskii Yu., Hydrodynamic time correlation functions of a Heisenberg ferrofluid. Physica A, 2000, 277, 389.

67. Folk R., Moser G., Critical dynamics of Heisenberg fluids at the gas-liquid transition. Phys. Rev. E, 2000, 61, 2864.

68. Folk R., Holovatch Yu., Yavors'kii T., Effective and asymptotic critical exponents of weakly diluted quenched Ising models: Three-dimensional approach versus $\sqrt{\varepsilon}$ expansion. Phys. Rev. B, 2000, 61, 15114.

69. Folk R., Critical transport and critical scattering in fluids. Condens. Matter Phys., 2000, 3, 359 .

70. Folk R., Holovatch Yu., Yavors'kii T., Pseudo- $\varepsilon$ expansion of six-loop renormalization group functions of an anisotropic cubic model. Phys. Rev. B, 2000, 62, 12195; Erratum: Phys. Rev. B, 2001, 63, 189901(E). 
71. Omelyan I.P., Mryglod I.M., Folk R., Spin dynamics simulations of collective excitations in magnetic liquids. Condens. Matter Phys., 2000, 2, 497.

72. Flossmann G., Folk R., Critical light scattering in liquids. Phys. Rev. E, 2000, 62, 2460.

73. Omelyan I.P., Mryglod I.M., Folk R., Algorithm for molecular dynamics simulations of spin liquids. Phys. Rev. Lett., 2001, 86, 898.

74. Omelyan I.P., Mryglod I.M., Folk R., Conservation laws preserving algorithms for spin dynamics simulations. Europhys. Lett., 2000, 52, 603.

75. Dudka M., Folk R., Holovatch Yu., On the critical behaviour of random anisotropy magnets. Condens. Matter Phys., 2001, 4, 77.

76. Mryglod I.M., Omelyan I.P., Folk R., Ferromagnetic phase transition in a Heisenberg fluid: Monte Carlo simulations and Fisher corrections to scaling. Phys. Rev. Lett., $2001,86,3156$.

77. Omelyan I.P., Mryglod I.M., Folk R., Molecular dynamics simulations of spin and pure liquids with preserving all the conservation laws. Phys. Rev. E, 2001, 64, 016105.

78. Mryglod I.M., Folk R., Corrections to scaling in systems with thermodynamic constraints. Physica A, 2001, 294, 351.

79. Folk R., Shopova D.V., Uzunov D.I., Fluctuation induced first order phase transition in thin films of type I superconductors. Phys. Lett. A, 2001, 281, 197.

80. Dudka M., Folk R., Holovatch Yu., On the critical behaviour of random anisotropy magnets: cubic anisotropy. Condens. Matter Phys., 2001, 4, 459.

81. Folk R., Holovatch Yu., Yavors'kii T., Critical exponents of a three dimensional weakly diluted quenched Ising model. Physics-Uspiekhi, 2003, 46, 169; Uspiekhi Fizichieskikh Nauk, 2003, 173, 175.

82. Omelyan I.P., Mryglod I.M., Folk R., Optimized Verlet-like algorithms for molecular dynamics simulations. Phys. Rev. E, 2002, 65, 056706.

83. Omelyan I.P., Mryglod I.M., Folk R., Optimized Forest-Ruth- and Suzuki-like algorithms for integration of motion in many-body systems. Computer Physics Communications, 2002, 146, 188.

84. Holovatch Yu., Blavats'ka V., Dudka M., von Ferber C., Folk R., Yavors'kii T., Weak quenched disorder and criticality: resummation of asymptotic(?) series. J. Mod. Phys. B, 2002, 16, 4027.

85. Omelyan I.P., Mryglod I.M., Folk R., Construction of high-order force-gradient algorithms for integration of motion in classical and quantum systems. Phys. Rev. E, 2002, 66, 026701.

86. Folk R., Moser G., Dynamic critical behavior near the superfluid transition in ${ }^{3} \mathrm{He}-$ ${ }^{4}$ He mixtures in two loop order. Phys. Rev. Lett., 2002, 89, 125301; Erratum: Phys. Rev. Lett., 2004, 93, 229902(E).

87. Dudka M., Folk R., Holovatch Yu., Ivaneiko D., Effective critical behaviour of diluted Heisenberg-like magnets. J. Magn. Magn. Mat., 2003, 256, 243. 
88. Omelyan I.P., Mryglod I.M., Folk R., Symplectic analytically integrable decomposition algorithms: classification, derivation, and application to molecular dynamics, quantum and celestial mechanics simulations. Computer Physics Communications, 2003, 151, 272.

89. Omelyan I.P., Mryglod I.M., Folk R., New optimized algorithms for molecular dynamics simulations. Condens. Matter Phys., 2002, 5, 369.

90. Mryglod I.M., Omelyan I.P., Folk R., Time correlation functions and dynamical properties of a Heisenberg fluid, J. Phys. Cond. Mat., 2003, 15, S83.

91. Fenz W., Folk R., Binary mixtures of magnetic fluids. Phys. Rev. E, 2003, 67, 021507.

92. Folk R., Moser G., Critical dynamics of model C resolved. Phys. Rev. Lett., 2003, 91, 030601.

93. Fenz W., Folk R., Mryglod I.M., Omelyan I.P., Phase diagrams of classical spin fluids: The influence of an external magnetic field on the liquid-gas transition, Phys. Rev. E, $2003,68,061510$.

94. Folk R., Moser G., Critical dynamics of stochastic models with energy conservation (model C). Phys. Rev. E, 2004, 69, 036101.

95. Fenz W., Folk R., Monte Carlo simulation of phase equilibria in Ising fluids and their mixtures. Condens. Matter Phys., 2003, 6, 675.

96. von Ferber C., Blavats'ka V., Folk R., Holovatch Yu., Where two fractals meet: the scaling of a self-avoiding walk on a percolation cluster. Phys. Rev. E, 2004, 70, 035104(R).

97. Omelyan I.P., Mryglod I.M., Folk R., Fenz W., Ising fluids in an external magnetic field: An integral equation approach. Phys. Rev. E, 2004, 69, 061506; Erratum: Phys. Rev. E, 2004, 70, 049903 (E).

98. Folk R., Moser G., Critical transport properties near the superfluid transition in ${ }^{3} \mathrm{He}-{ }^{4} \mathrm{He}$ mixtures. J. Low Temp. Phys., 2004, 136, 159.

99. Omelyan I.P., Fenz W., Mryglod I.M., Folk R., XY-spin fluid in an external magnetic field. Phys. Rev. Lett., 2005, 94, 045701.

100. Folk R., Moser G., The critical dynamics of stochastic models with two conserved densities (model C'). Phys. Rev. E, 2005, 71, 026118. 